\title{
Triple Intrathecal Combination Therapy for End-Stage Cancer-Related Refractory Pain: A Prospective Observational Study with Two-Month Follow-Up
}

\author{
Filomena Puntillo · Mariateresa Giglio · Angela Preziosa • \\ Lidia Dalfino · Francesco Bruno · Nicola Brienza - Giustino Varrassi
}

Received: March 10, 2020 / Published online: April 29, 2020

(C) The Author(s) 2020

\begin{abstract}
Introduction: In cancer-related pain refractory to systemic opioids, intrathecal (IT) administration of morphine can be a useful strategy. In clinical practice, IT morphine is usually combined with other drugs with different mechanisms of action, in order to obtain a synergistic analgesic effect. However, the discussion on efficacy and safety of IT combination therapy is still ongoing. The aim of this observational study was to report the effects of an IT combination of low doses of ziconotide, morphine, and levobupivacaine in end-stage cancer refractory pain.
\end{abstract}

Enhanced Digital Features To view digital features for this article go to https://doi.org/10.6084/m9.figshare. 12136251.

F. Puntillo $(\bowtie)$

Department of Interdisciplinary Medicine,

University of Bari "Aldo Moro", Bari, Italy

e-mail: filomena.puntillo@uniba.it;

nuccia.puntillo@gmail.com

M. Giglio · A. Preziosa - L. Dalfino

Anaesthesia and Intensive Care Unit, Policlinico

Hospital, Bari, Italy

F. Bruno $\cdot$ N. Brienza

Department of Emergency and Organ

Transplantation, University of Bari "Aldo Moro",

Bari, Italy

G. Varrassi

Paolo Procacci Foundation, Rome, Italy
Methods: Sixty adult patients, 21 females and 39 males, were enrolled to an IT device implant. The mean visual analogue scale of pain intensity (VASPI) score was $88 \pm 20 \mathrm{~mm}$. All patients started with a triple combination therapy: the initial IT dose of morphine was calculated for each patient based on the equivalent daily dose of morphine; an oral/IT ratio of 400/1 was used. For ziconotide, a standard slow titration schedule was started at $1.2 \mu \mathrm{g} /$ day and the initial dose of levobupivacaine was $3 \mathrm{mg} /$ day.

Results: The initial IT mean doses of morphine, ziconotide, and levobupivacaine were $0.8 \pm 0.3 \mathrm{mg} / \mathrm{day}, 1.2 \mathrm{mcg} /$ day and $3 \mathrm{mg} /$ day, respectively. At day 2 , a significant reduction in VASPI score was registered $(49 \pm 17, p<0.001)$, and this significant reduction persisted at 56 days (mean VASPI score $44 \pm 9, p<0.001$ ), with mean doses of morphine $2 \pm 1 \mathrm{mg}$ /day, ziconotide $2.8 \pm 1 \mathrm{mcg} /$ day, and levobupivacaine $3.8 \pm 2 \mathrm{mg} /$ day. Very few adverse effects (AEs) were observed. Patients' satisfaction was very high during the entire study period.

Conclusions: Our results, within the limit of the study design, suggest that the IT combination of ziconotide, morphine, and levobupivacaine, at low doses, allows safe and rapid control of refractory cancer pain, with high levels of patient satisfaction.

Keywords: Cancer pain; Drug therapy combination; Intrathecal; Levobupivacaine; 
Morphine; Patient satisfaction; Visual analogue pain scale; Ziconotide

\section{Key Summary Points}

End-stage cancer pain is still a challenge, and sometimes requires interventional strategies to control it.

Intrathecal ziconotide can have a synergistic effect with morphine controlling neuropathic pain, since it gives a more complete blockade of synaptic transmission from cells bearing N-type calcium channels.

Adding levobupivacaine (that blocks voltage-gated sodium channels on $\mathrm{C}$ and Ad fibers in primary afferents), to ziconotide and morphine can be a winning choice, assuring better analgesia and patient satisfaction, with low doses of three drugs.

Triple intrathecal combination therapy through a subcutaneous port-a-cath can be a low-invasive efficient strategy to control pain in end-stage, difficult-to-treat cancer patients.

\section{INTRODUCTION}

The prevalence of cancer-related pain has been reported to be $30-40 \%$ in the early stage of the disease. It increases to $70-90 \%$ in the advanced stage [1]. Chronic pain after cure of cancer may be as high as 33\% [2]. Analgesic prescriptions according to the World Health Organization ladder are effective in over $80 \%$ of cases. Nevertheless, in $15-20 \%$ of patients, conventional analgesic therapy either fails to relieve pain or induces unacceptable adverse events [3]. A fourth step consisting of interventional analgesic techniques such as radiotherapy, interventional radiology, surgery, and epidural as well as intrathecal (IT) analgesia has been suggested by Miguel [4]. Spinal analgesia may provide a useful means to control pain in such difficult situations because the administration of small amounts of opioids in close proximity to their spinal receptors would achieve high concentrations at these sites, resulting in a superior analgesia, reducing adverse effects (AEs) [5]. However, because of the difficulty to treat cancer-related pain, higher doses, higher concentrations, or combinations of drugs that are out-label may be required to control pain [6].

A previous study suggests that IT combination of ziconotide and morphine, at low doses, allows a safe and rapid control of oral opioids refractory malignant pain [7]. Several cases of evidence also suggested a synergistic effect of morphine and local anesthetic, such as bupivacaine $[8,9]$, even if a more recent trial [10] suggested that IT analgesia using bupivacaine is not enhanced by coadministration of morphine in patients with severe cancer-related pain.

The rationale to combine ziconotide and morphine is that although they share the same target on presynaptic neurons (i.e., N-type calcium channels), morphine acts on both presynaptic and postsynaptic neurons and on other molecular targets in nonoverlapping subsets of neurons. Hence, the addition of ziconotide to an IT opioid would result in more complete blockade of synaptic transmission from cells bearing N-type calcium channels. On the other side, local anesthetics primarily block voltagegated sodium channels on $\mathrm{C}$ and Ad fibers in primary afferents, disrupting propagation of the action potential from the periphery into the DRG and dorsal horn [11].

The aim of this study is to report the effect (i.e., pain reduction, AEs and patient satisfaction) of IT combination of ziconotide, morphine, and levobupivacaine at low doses in endstage cancer-related pain refractory to high doses of oral/transdermal opioids.

\section{METHODS}

This prospective observational study was conducted according to the ethical principles of the current amended version of the Helsinki Declaration and IASP's guidelines for pain research 
in humans. Approval from the Ethical Committee of Policlinico Hospital, Bari was obtained (cod ITZML001) and each patient gave signed informed consent. The study took place in the Pain Center of Policlinico Hospital, Bari, from 2015 to 2018.

Eligible patients were at least 18 years of age with severe chronic pain attributable to endstage cancer in which IT therapy was recommended because of inefficacy or intolerance to strong systemic opioid treatment. Inefficacy was defined as a VASPI score $(0-100 \mathrm{~mm}$, with $0 \mathrm{~mm}$ representing no pain and $100 \mathrm{~mm}$ representing the worst pain imaginable) at rest $>70 \mathrm{~mm}$ although strong systemic opioid treatment (more than $200 \mathrm{mg} /$ day of oral morphine equivalents). Intolerance was defined as the occurrence of severe AEs (even with dosage less than $200 \mathrm{mg} /$ day of oral morphine equivalents) which prevents a further increase in the opioid dosage to obtain pain relief. More than one opioid rotation had to be done before defining inefficacy and intolerance. Patients were excluded if they had signs of sepsis or inadequately treated infection, uncontrolled heart failure or second-third-degree heart block, history of dementia, delirium, hysteria, or an untreated affective disorder.

At the first visit, medical history, concomitant medications, at rest and incident VASPI score of the previous week, and the Karnofsky Performance Status Score (KPSS) were recorded for each patient. After enrolling, the implant of an IT catheter was planned. The procedure was performed under fluoroscopy and the catheter was connected to an external controlled infusion system through a subcutaneous port-a-cath to reduce the risk of catheter infection. A shortterm antibiotic intravenous therapy (cefazolin $1 \mathrm{~g}$ ) was administered. The tip of the catheter was placed near dermatomal level of the worst pain, considering the limited capacity for cerebrospinal fluid to distribute drugs away from the catheter tip [12]. The patients were asked to continue long-acting oral or transdermal opioids until the day of the implant. IT infusion without bolus was used in order to achieve the IT target dose very slowly, allowing a safe overlap with the last dose of long-acting opioid. After the IT infusion was started, the patients were asked to stop their previous opioid therapy and to assume a short-acting oral morphine dose $(30 \mathrm{mg})$ as rescue medication even more times a day, if their background pain was poorly controlled. Patients that were already assuming rapid onset opioids for breakthrough pain were also asked to continue, as needed. An IT combination therapy with ziconotide, levobupivacaine, and morphine was performed: for ziconotide a standard slow titration schedule was started at a minimum dose of $1.2 \mu \mathrm{g}$ /day followed by increases of $1.2 \mu \mathrm{g} /$ day at intervals of 7 days; in case of hallucinations, dizziness, or aggressivity, ziconotide was reduced of $50 \%$ or stopped, depending on the severity of symptoms. The initial IT dose of morphine was calculated for each patient based on the equivalent daily dose of morphine; an oral/IT ratio of 400/1 was used. At each control (every 7 days) an increase of daily dose of IT morphine was allowed considering the amount of short-acting oral morphine consumption. The initial dose of levobupivacaine was $3 \mathrm{mg} /$ day. At each control, an increase of $50-70 \%$ in the daily dose of levobupivacaine was allowed, based on the presence of neuropathic pain signs; in case of evidence of leg weakness or urinary retention the dose was no more increased.

No maximum dose limit was defined for morphine. For ziconotide and levobupivacaine, the maximum dose was fixed according to the Polyanalgesic Consensus Conference [13].

Primary outcome was the reduction of VASPI score over the weeks. Secondary outcomes were evaluation of AEs rate and the satisfaction of the patients measured by a five-point scale. Patients rated their satisfaction as "very much satisfied (4)," "much satisfied (3)," "satisfied (2) ", "minimally satisfied (1)", "not satisfied (0)".

After 4 weeks of treatment, in patients presumed to have a life expectancy of more than 3 months, the usefulness of an implantable system (SynchroMed ${ }^{\circledR}$ Infusion System, Medtronic, Inc., Minneapolis, MN, USA) was evaluated in order to reduce the risk of meningitidis [14] and the discomfort of an external device. The increased invasiveness of an internal pump implant and patient preference were also taken into consideration before to implant it. Life span was evaluated by a multiprofessional team 
(i.e., oncologists, pain and palliative physicians), as this may help refine the prognostic estimate [15].

\section{Efficacy and Safety Measurements}

VASPI score, vital signs (including systolic and diastolic blood pressure, heart rate, respiratory rate and body temperature), a 12-lead electrocardiogram (ECG) and AEs were evaluated at 2 days, at 7 days, and then weekly until the end of treatment. At day 2, the mean VASPI score of the last $48 \mathrm{~h}$ was registered, while at 7 days and weekly the mean VASPI score of the last 7 days was collected. Only two experienced nurses collected the data. All AEs were coded with the Coding Symbols for Thesaurus of Adverse Reaction Terms, Fifth Edition, Dictionary. For each $\mathrm{AE}$, the investigator determined the severity, the relationship to every study drug, and if the AE was serious or nonserious. Serious AEs are those that were fatal, immediately lifethreatening, or significantly disabling. Laboratory evaluations for serum creatine kinase (CK) levels were performed at initial visit and monthly.

\section{Statistical Analysis}

Mean VASPI score, mean change, and mean percentage change in VASPI score from baseline to each visit and to the last available observation were calculated. Two-sides $95 \%$ confidence intervals were calculated for each mean change and percentage change in VASPI score at $48 \mathrm{~h}$, at week 1 , and weekly until the end of treatment were also calculated; at the same time, the mean change and percentage change were compared to zero using a non-parametric oneway ANOVA. The null hypothesis, which states that the mean change (or percentage change) is not different from zero, was tested in each case. Changes from baseline for vital signs and ECG readings were analyzed with paired $t$ test. All tests were two-tailed with a $\alpha$ level $<0.05$ considered statistically significant. The tests were performed with the program SPSS version 12.0 for Windows.

\section{RESULTS}

During the study period, 64 patients were considered potentially eligible. Of these, four were excluded (two for inadequately treated infection, and two for untreated affective disorder). In total, 60 patients, 21 females and 39 males, were enrolled. Their mean age was $63 \pm 12$. All had advanced cancer with metastasis, $75 \%$ of patients had bone metastases. The mean daily VASPI score at rest was $88 \pm 6 \mathrm{~mm}$, the incident VASPI score was $100 \mathrm{~mm}$. The mean KPSS was $54 \pm 15$. Table 1 depicts demographic data of the patients and the distribution of the different types of carcinomas.

Before enrollment, all patients were treated with high doses of long-acting oral or transdermal opioids (mean dose $240 \pm 80 \mathrm{mg}$ of morphine equivalents) but all of them had a poorly controlled pain and/or experienced adverse events related to high doses of opioids. On average, life expectancy was lower than 3 months.

Table 1 Demographic characteristics of the included patients and carcinoma's distribution

\begin{tabular}{ll}
\hline & $n=\mathbf{6 0}$ \\
\hline Age (mean \pm DS) & $63 \pm 12$ \\
$F / M$ & $21 / 39$ \\
Pancreatic Cr & 9 \\
Urotelial Cr & 7 \\
Mammalian Cr & 4 \\
Gastrointestinal Cr & 15 \\
Lung Cr & 17 \\
Hepatic Cr & 1 \\
Melanoma & 2 \\
Uterus Cr & 3 \\
Tongue Cr & 2 \\
Days on IT (range) & $10-175$ \\
VASPI at rest (mean \pm DS) & $88 \pm 6$ \\
\hline
\end{tabular}


The initial IT mean daily doses were $0.8 \pm 0.3 \mathrm{mg}$ for morphine, $1.2 \mathrm{mcg}$ for ziconotide and $3 \mathrm{mg}$ for levobupivacaine. At day 2, a significant reduction in VASPI score was registered (49 $\pm 17, p<0.001)$, and this significant reduction persisted at 56 days (mean VASPI score $44 \pm 9, p<0.001$ ), when the mean daily doses were $2 \pm 1 \mathrm{mg}$ for morphine, $2.8 \pm 1 \mathrm{mcg}$ for ziconotide, and $3.8 \pm 2 \mathrm{mg}$ for levobupivacaine. Figure 1 depicts the trend of VASPI score at every observation time. Table 2 shows the values of VASPI and mean VASPI changes over time.

Figure 2 shows the trend of mean daily dose of each drug from baseline at every observation time. The maximum mean dose of morphine was $2 \mathrm{mg} / \mathrm{die}$, the maximum daily dose of ziconotide was $2.8 \mathrm{mcg} /$ day and the maximum mean daily dose of levobupivacaine was $4.4 \mathrm{mg} /$ day. All patients stopped their previous systemic opioid therapy and also the use of gabapentinoids; $70 \%$ of patients reported a sporadic intake of $30 \mathrm{mg}$ of short acting oral morphine, $30 \%$ of patients continued to intake only acetaminophen as needed. Six patients reported use of rapid onset opioids to control breakthrough pain (range $400-600 \mathrm{mcg}$ of transmucosal fentanyl), for no more than two times a day.

The incidence of any AE ranged from 3.3 to $10 \%$. Out of 60 patients, five (8.3\%) experienced dizziness, six (10\%) confusion, six (10\%) reported urinary retention, six $(10 \%)$ nausea, and three (5\%) vomiting, two (3.3\%) aggressivity, and two (3.3\%) hallucinations. No leg anesthesia or leg weakness were reported. No increase in CPK and no hemodynamic AEs were observed. Only one, high-risk, HIV patient explanted the IT infusion system due to a pocket infection of the port.

In six patients (10\%), the dose of ziconotide was reduced due to hallucinations and dizziness, in four patients (6\%) ziconotide was stopped because of aggressivity. In all of these

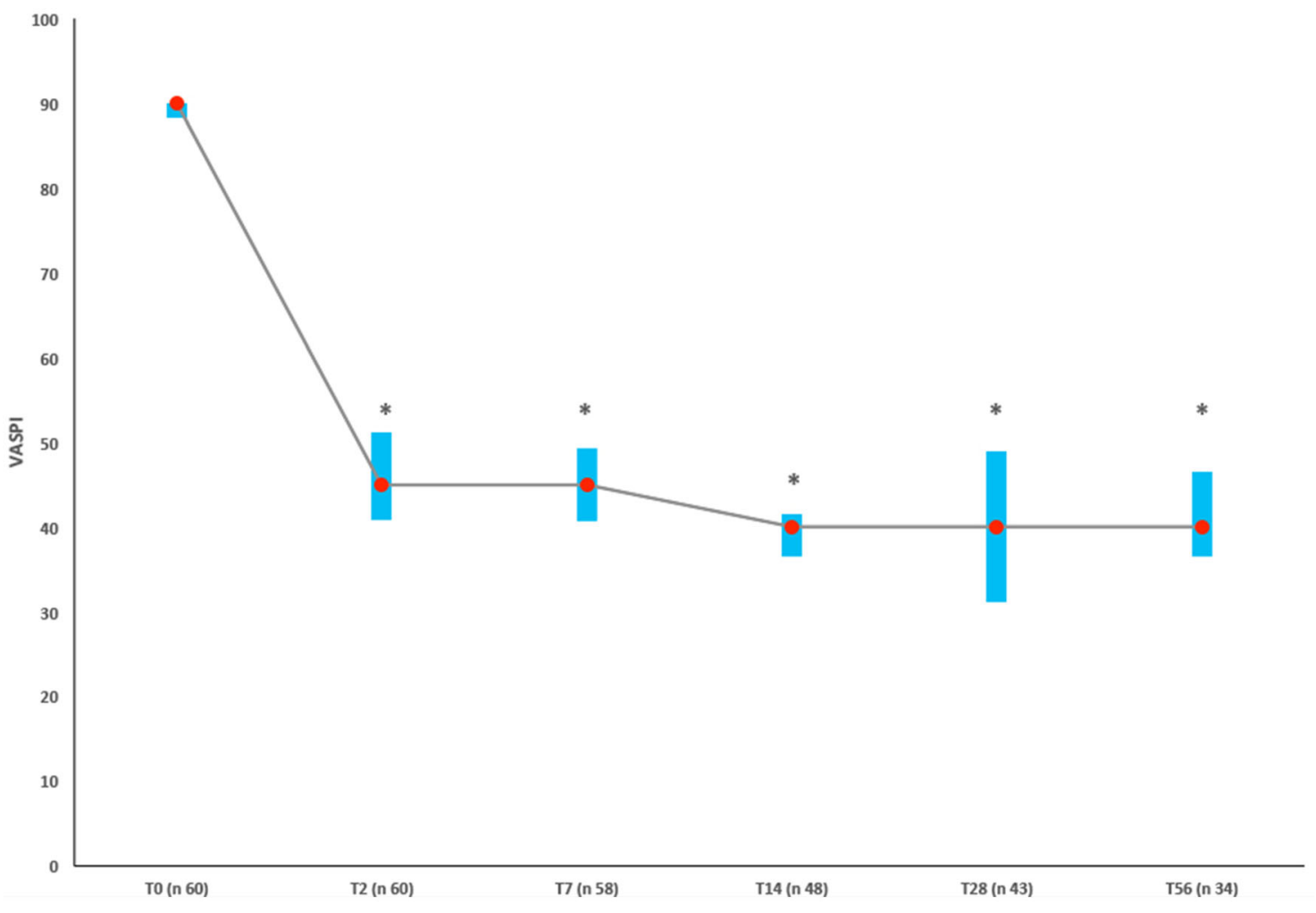

Fig. 1 The reduction of VASPI score (median) over time ${ }^{*} p<0.05$. ( $T 0=$ day of IT catheter placement, $T 2=2$ days, $T 7=7$ days, $T 14=14$ days and so on). $n$ Sample size 
Table 2 VASPI score at rest, mean VASPI change, and 95\% CI (confidence interval) over time from day $0(T 0)$ to day 56 (T56)

\begin{tabular}{|c|c|c|c|c|c|c|}
\hline & $\begin{array}{l}T 0 \\
(n=60)\end{array}$ & $\begin{array}{l}T 2 \\
(n=60)\end{array}$ & $\begin{array}{l}T 7 \\
(n=60)\end{array}$ & $\begin{array}{l}T 14 \\
(n=58)\end{array}$ & $\begin{array}{l}T 28 \\
(n=48)\end{array}$ & $\begin{array}{l}T 56 \\
(n=35)\end{array}$ \\
\hline VASPI at rest mean $\pm S D(\mathrm{~mm})$ & $88 \pm 6$ & $49 \pm 17$ & $48 \pm 16$ & $44 \pm 16$ & $43 \pm 14$ & $44 \pm 9$ \\
\hline $\begin{array}{l}\text { Mean VASPI change from } T 0 \pm S D \\
(\mathrm{~mm})\end{array}$ & & $39 \pm 20$ & $40 \pm 20$ & $45 \pm 18$ & $53 \pm 18$ & $53 \pm 8$ \\
\hline $95 \%$ CI of mean VASPI change $(\mathrm{mm})$ & & $34-44$ & $35-45$ & $41-50$ & $49-59$ & $51-56$ \\
\hline$p$ value & & $<0.05$ & $<0.05$ & $<0.05$ & $<0.05$ & $<0.05$ \\
\hline
\end{tabular}

$n$ Sample size

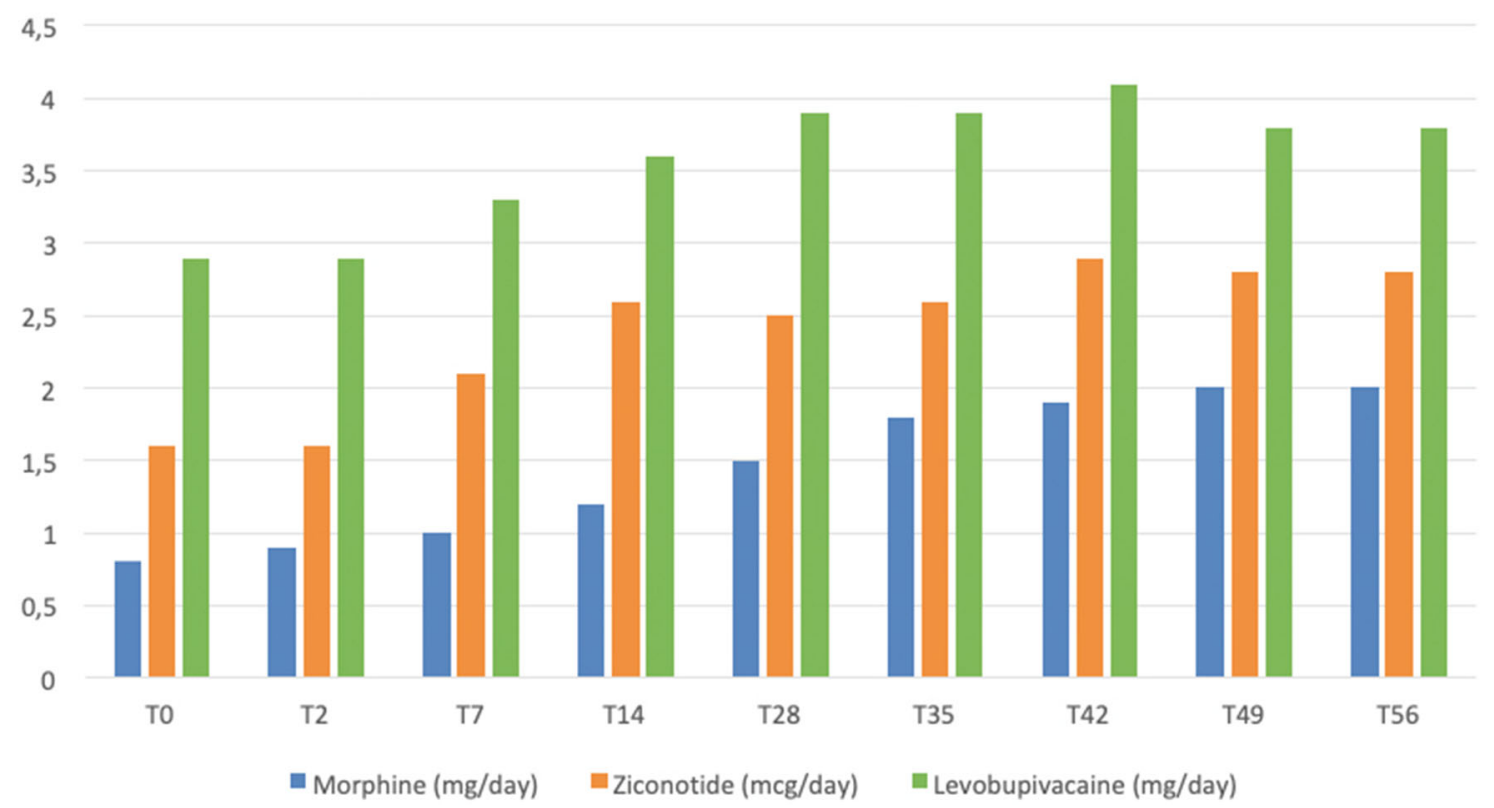

Fig. 2 The mean daily dosage of three drugs over time from day 0 to day 56

ten patients, an increase greater than 30\% in the daily doses of morphine and levobupivacaine was deemed necessary to maintain the control of pain in the following weeks.

Patient satisfaction scores showed high levels of satisfaction from T7 lasting for the entire duration of the study (see Fig. 3).

Two patients (both with pelvic cancer) underwent an internal pump implant after the first month of treatment.

\section{DISCUSSION}

The current study suggests that a triple IT therapy with ziconotide, morphine, and levobupivacaine is a successful strategy in endstage patients with malignant pain refractory to high doses of systemic opioids. This combination allows rapid control of pain, as demonstrated by the significant reduction of VASPI score as soon as 2 days. The addition of levobupivacaine seems to potentiate the effects of the other two drugs, allowing a reduction in 


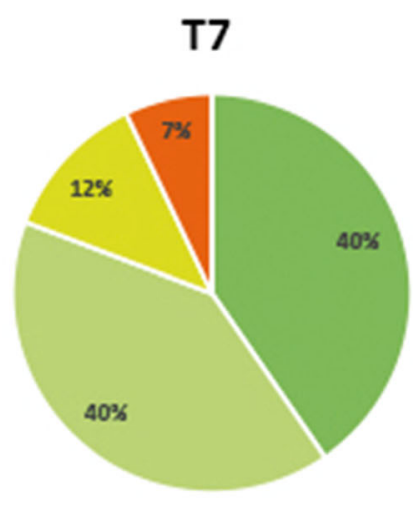

T28

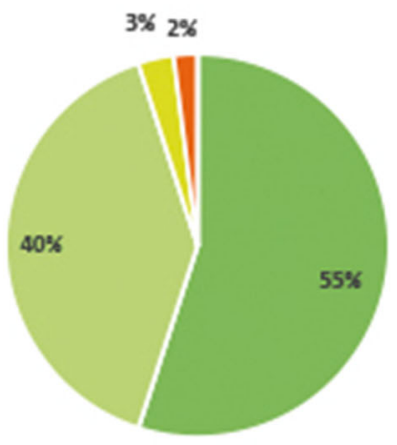

" very much satisfied

much satisfied

satisfied

= minimally satisfied

not satisfied
T14
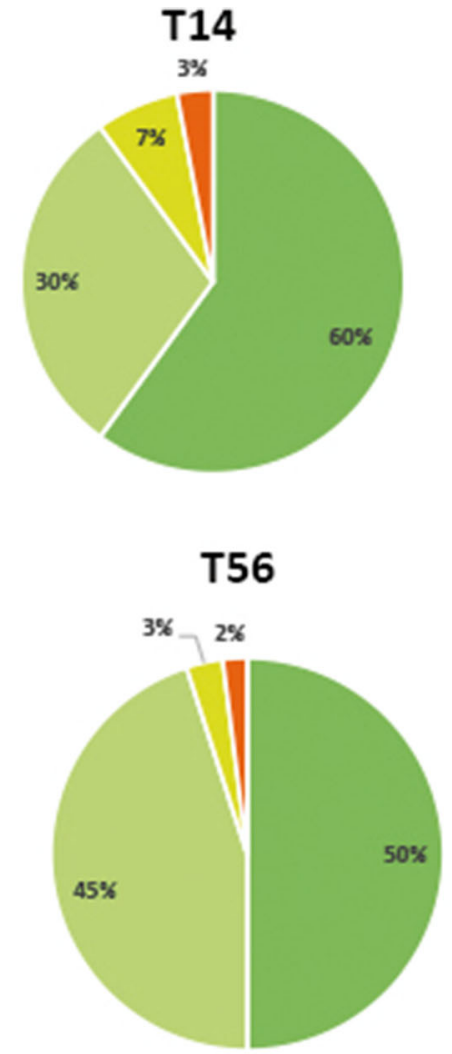

Fig. 3 Patient satisfaction rated as-very much satisfied (4)," "much satisfied (3)," "satisfied (2) ”, "minimally satisfied (1)”, “not satisfied (0)" —at $T 7$ (7 days), T14 (14 days) at T28 (28 days), T56 (56 days)

cumulative doses of both morphine and ziconotide over time, compared to those reported in similar populations $[7,16]$. Of note, in the present trial, both the starting dose and the maximum dose of ziconotide were lower than previously reported [7], suggesting that levobupivacaine can have a synergistic, sparing effect also in combination with ziconotide. Finally, in all our patients who required ziconotide reduction and/or suspension due to AEs, an increase in the daily doses of morphine and levobupivacaine was necessary to maintain similar VASPI scores.

IT multimodal therapy is based on the principle that combining drugs with different mechanisms of action could produce synergistic effect on pain reduction, spare doses of each drug and reduce AEs. Notably, morphine and ziconotide are the only two agents approved in for IT administration [13]. The use of other agents (i.e., local anesthetics), although not suggested as first-line therapy, is common among pain physicians who use IT pumps, even if discussions on efficacy and patient safety of such agents is still ongoing. Among local anesthetics, levobupivacaine, compared to bupivacaine, has less neurotoxic and cardiotoxic effect, as ropivacaine does, but it is more potent and produces a longer effect in comparison to ropivacaine [17]. There are evidences that a combination of morphine and levobupivacaine for highly refractory cancer-related pain significantly decreases mean pain intensity and systemic opioid consumption, with mild AEs $[18,19]$. On the other side, it has been suggested that ziconotide in combination with local anesthetics reduces morphine consumption with acceptable safety profile in acute postoperative pain [20]. Our study suggests that an IT combination therapy of morphine, ziconotide, and levobupivacaine assures a significant, rapid and stable control of cancer-related pain, as the 
statistical reduction of VASPI scores from 2 days to 2 months of observation demonstrates, with little doses of all three drugs. All these doses were reached with low increases every week, confirming the beneficial effect of the combination on pain reduction and on the sparing effect of the total daily doses of the three drugs. When a single drug IT infusion was adopted, pain control was obtained with higher daily doses both for morphine [21] and for ziconotide [22]. Of note, our maximum daily dose of IT morphine was below the recent suggested cut off [23] that assures undetectable serum concentration of morphine, thus allowing reduced systemic toxicity. Moreover, $70 \%$ of patients reported sporadic intake of oral morphine, thus confirming the sparing effect of the combination on the systemic daily dose of morphine, even in these end-stage cancer patients in which pain intensity is assumed to increase over the week according to cancer progression.

The careful and slow titration over time explains why only few patients developed AEs, even if we registered a higher incidence of urinary retention (10\%) compared to our first trial [7]. Many factors could explain this finding, including the presence of several patients with pelvic cancer. Maybe levobupivacaine could potentiate the effect on urinary function of the other two drugs even if only two patients needed urinary catheterization. However, when an IT association of morphine, ziconotide, and local anesthetic is planned to treat patients with pelvic cancer, this potential adverse effect should be considered.

Furthermore, when an IT therapy combination is given, it is important to consider drug stability, as drugs with low chemical stabilities may require more frequent pump refills. Opioids can speed ziconotide degradation, reducing its stability, even if low morphine concentration $(20 \mathrm{mg} / \mathrm{ml})$ assures longer ziconotide stability (up to 19 days) [24]. Also, bupivacaine (range of mean concentrations $0.9-3.1 \%$ ), coformulated with morphine (mean concentration $2.6 \%$ ) was chemically stable during IT infusion for periods between 2 and 7 weeks [25]. In this study, we used low morphine concentrations and weekly pump refills, thus ensuring stable ziconotide concentrations. In addition, the slow infusion rate and the low concentrations used could account for the low incidence of AEs.

Patient satisfaction was surprising. Although they were afraid of the invasiveness of the treatment, and of the limitation of the mobility due to the external pump, the good pain control has overcome all their fears. A careful weekly pain check probably contributed to this result.

The present observational study has some limitations related to the study design, (single arm study), the small number of patients included due to the very difficult-to-treat category of patients enrolled. The non-randomized nature of the study does not permit to reach firm conclusions, nor to clearly explore the synergistic effect of the association. The short life expectancy due to cancer progression limited the time of observation to 2 months, as only 13 patients survived after the third month. However, this strategy suggests rapid and efficacious pain control and calls for more studies enrolling a larger population of oncological patients with refractory pain.

\section{CONCLUSIONS}

Our study, within the limit of the study design, suggests that a triple IT combination of ziconotide, morphine, and levobupivacaine, at low doses, allows safe and rapid control of oral opioid-refractory malignant pain, with high levels of patient satisfaction. The decrease in VASPI score was significant within 2 days after the IT combination was initiated and persisted during the 56 days of the study. Mild AEs were observed in few patients.

\section{ACKNOWLEDGEMENTS}

The authors express sincere gratitude to all of the staff members of the Pain Center at Policlinico Hospital, Bari, Italy, for their precious contribution. We also thank the participants of the study. 
Funding. The study was conducted only by department resources. No Rapid Service Fee was received by the journal for the publication of this article.

Authorship. All named authors meet the International Committee of Medical Journal Editors (ICMJE) criteria for authorship for this article, take responsibility for the integrity of the work as a whole, and have given their approval for this version to be published.

Authorship Contributions. All authors carried out most of the selection of the articles and drafted the initial manuscript. All the authors contributed to review and critical revision to improve the quality of the paper. All authors have reviewed and approved the final draft of the submitted manuscript.

Disclosures. Mariateresa Giglio, Angela Preziosa, Lidia Dalfino, Nicola Brienza, Francesco Bruno have nothing to disclose. Giustino Varrassi and Filomena Puntillo are members of the journal's Editorial Board.

Compliance with Ethics Guidelines. This prospective observational study was conducted according to the ethical principles of the current amended version of the Helsinki Declaration and IASP's guidelines for pain research in humans. Approval from the Ethical Committee of Policlinico Hospital, Bari was obtained (cod ITZML001) and each patient gave signed informed consent.

Data Availability. The datasets during and/ or analyzed during the current study are available from the corresponding author on reasonable request.

Open Access. This article is licensed under a Creative Commons Attribution-NonCommercial 4.0 International License, which permits any non-commercial use, sharing, adaptation, distribution and reproduction in any medium or format, as long as you give appropriate credit to the original author(s) and the source, provide a link to the Creative Commons licence, and indicate if changes were made. The images or other third party material in this article are included in the article's Creative Commons licence, unless indicated otherwise in a credit line to the material. If material is not included in the article's Creative Commons licence and your intended use is not permitted by statutory regulation or exceeds the permitted use, you will need to obtain permission directly from the copyright holder. To view a copy of this licence, visit http://creativecommons.org/licenses/by$\mathrm{nc} / 4.0 /$.

\section{REFERENCES}

1. Foley KM. Acute and chronic cancer pain syndromes. In: Doyle D, Hanks G, Cherny N, Calman $\mathrm{K}$, editors. Oxford textbook of palliative medicine. 3rd ed. Oxford: Oxford University Press; 2004. p. 298-316.

2. van den Beuken-van Everdingen $\mathrm{MH}$, de Rijke JM, Kessels AG, Schouten HC, van Kleef M, Patijn J. Prevalence of pain in patients with cancer: a systematic review of the past 40 years. Ann Oncol. 2007;18:1437-49.

3. Zech DF, Grond S, Meuser T, et al. Symptoms during cancer pain treatment following WHO-guidelines: a longitudinal follow-up study of symptom prevalence, severity and etiology. Pain. 2001;93: 247-57.

4. Miguel R. Interventional treatment of cancer pain: the fourth step in the World Health Organization analgesic ladder? Cancer Control. 2000;7:149-56.

5. Mercadante S, Porzio G, Gebbia V. Spinal analgesia for advanced cancer patients: an update. Crit Rev Oncol Hematol. 2012;82:227-32.

6. Deer TR, Prager J, Levy R, et al. Polyanalgesic Consensus Conference 2012: recommendations for the management of pain by intrathecal (intraspinal) drug delivery: report of an interdisciplinary expert panel. Neuromodulation. 2012;15:436-66.

7. Alicino I, Giglio M, Manca F, Bruno F, Puntillo F. Intrathecal combination of ziconotide and morphine for refractory malignant pain: a rapid and effective choice. Pain. 2012;153:245-9.

8. van Dongen R, Crul B, van Egmond J. Intrathecal coadministration of bupivacaine diminishes morphine dose progression during long-term intrathecal infusion in cancer patients. Clin J Pain. 1999;15: 166-72. 
9. Deer TR, Caraway DL, Kim CK, Dempsey CD, Stewart $\mathrm{CD}$, McNeil KF. Clinical experience with intrathecal bupivacaine in combination with opioid for the treatment of chronic pain related to failed back surgery syndrome and metastatic cancer pain of the spine. Spine J. 2002;2:274-8.

10. Reif I, Wincent A, Stiller CO. Intrathecal analgesia by bupivacaine is not enhanced by coadministration of morphine in patients with severe cancerrelated pain: a randomized double-blind cross-over study. Int J Clin Pharmacol Ther. 2017;55:525-32.

11. Miljanich G, Rauck R, Saulino M. Spinal mechanisms of pain and analgesia. Pain Pract. 2013;1: 14-130.

12. Bernards CM. Cerebrospinal fluid and spinal cord distribution of baclofen and bupivacaine during slow intrathecal infusion in pigs. Anesthesiology. 2006;105:169-78.

13. Deer T, Pope JE, Hayek SM, et al. The Polyanalgesic Consensus Conference (PACC): recommendations on intrathecal drug infusion systems best practices and guidelines. Neuromodulation. 2017;20:96-132.

14. Kress HG, Simpson KH, Marchettini P, Ver Donck A, Varrassi G. Intrathecal therapy: what has changed with the introduction of ziconotide. Pain Pract. 2009;9:338-47.

15. Chu C, Anderson R, White N, Stone P. Prognosticating for adult patients with advanced incurable cancer: a needed oncologist skill. Curr Treat Opt Oncol. 2020;21(1):5.

16. Dupoiron D, Bore F, Lefebvre-Kuntz D, Brenet O, Debourmont $\mathrm{S}$, et al. Ziconotide adverse events in patients with cancer pain: a multicenter observational study of a slow titration, multidrug protocol. Pain Phys. 2012;15:395-403.

17. Foster RH, Markham A. Levobupivacaine: a review of its pharmacology and use as a local anaesthetic. Drugs. 2000;59:551-79.
18. Mercadante $S$, Intravaia $G$, Villari $P$, et al. Intrathecal treatment in cancer patients unresponsive to multiple trials of systemic opioids. Clin J Pain. 2007;23:793-8.

19. Mitchell A, McGhie J, Owen M, McGinn G. Audit of intrathecal drug delivery for patients with difficultto-control cancer pain shows a sustained reduction in pain severity scores over a 6-month period. Palliat Med. 2015;29:554-63.

20. Atanassoff PG, Hartmannsgruber MW, Thrasher J, et al. Ziconotide, a new N-type calcium channel blocker, administered intrathecally for acute postoperative pain. Reg Anesth Pain Med. 2000;25: 274-8.

21. Rauck RL, Cherry D, Boyer MF, Kosek P, Dunn J, Alo K. Long-term intrathecal opioid therapy with a patient-activated, implanted delivery system for the treatment of refractory cancer pain. J Pain. 2003;4(8):441-7.

22. Raffaeli W, Sarti D, Demartini L, Sotgiu A, Bonezzi C, Italian Ziconotide Group. Italian registry on long-term intrathecal ziconotide treatment. Physician. 2011;14:15-24.

23. Brogan SE, Sindt JE, Jackman CM, White J, Wilding $\mathrm{V}$, Okifuji A. A prospective association of serum opioid levels and clinical outcomes in patients with cancer pain treated with intrathecal opioid therapy. Anest Analg. 2019;130(4):1035-44.

24. Dupoiron D, Richard H, Chabert-Desnot V, Devys C, Leynia P, Boisdron-Celle M. In vitro stability of low-concentration ziconotide alone or in admixtures in intrathecal pumps. Neuromodulation. 2014;17:472-82.

25. Goucke CR, Dusci LJ, Van Leeuwen S, Fairclough D, Ilett KF. Stability and tolerability of high concentrations of intrathecal bupivacaine and opioid mixtures in chronic noncancer pain: an open-label pilot study. Pain Med. 2010;11:1612-8. 